\title{
MODELLING THE RELATIONSHIP BETWEEN THE LEVEL OF ADOPTION OF SUSTAINABILITY PRACTICES AND PERFORMANCE OF CONSTRUCTION FIRMS USING STRUCTURAL EQUATION MODELLING
}

\author{
Monday OTALI' ${ }^{1}$, Godwin IDORO², Godfey UDO $^{3}$ \\ ${ }^{1,3}$ University of Uyo, Uyo, Nigeria \\ ${ }^{2}$ University of Lagos, Lagos, Nigeria \\ Corresponding author's e-mail:otalimonday@yahoo.com
}

\begin{abstract}
The need to carry out construction activities in a sustainable manner in the Niger Delta region of Nigeria has become very imperative. The aim of the study was to establish the relationship between the adoption of sustainable construction practices and performance of construction firms using a structural equation modelling approach. In tandem with the survey approach adopted for the study, 1179 copies of structured questionnaire were administered while 980 valid responses were received giving a response rate of $83.2 \%$. Data were analysed using descriptive statistics and structural equation modelling. This study revealed that the extent of implementation of sustainable construction practices accounted for $36 \%, 37.8 \%, 43.5 \%, 51.6 \%, 65.8 \%$, and $32.9 \%$ of the variability in annual employment growth, annual financial turnover, remuneration and benefits, general employee satisfaction, social performance and net income growth of the construction firms, respectively. The study concluded that there was a positive relationship between the extent of implementation of sustainable construction practices and firm performance. It was also concluded that the adoption level of sustainable construction practices accounted for $85 \%$ of the variability in the overall firm performance. Hence, proper implementation of sustainability practices at the firm level leads to improvement in the firm performance. It was recommended that construction firms should not use only financial performance indicators to evaluate their performance level but rather use multi-dimensional approaches. This will enable firms to have holistic knowledge of the level of their performance. The construction firms should adopt the structural equation model developed in the study to carry out integrated performance measurement.
\end{abstract}

Keywords: level of adoption; Niger Delta; performance of construction firms; relationship; structural equation modelling; sustainability practices.

\section{INTRODUCTION}

There is a growing pressure to develop the environment in a sustainable manner. This includes environmental, social and economic sustainability. The building industry is responsible for infrastructural development of the society but it consumes a lot of resources and energy. In addition, it is responsible for pollution, 
climate change and other environmental hazards (Klang, Vikman and Brattebo, 2003).

Corporate-level sustainability is the ability to achieve social and environmental needs, as well as company profitability (Porter, 2008). Brundtland (1987) defined sustainable development as that which meets the needs of the present generation without jeopardizing the chances of the future generations to meet their needs. Sustainable construction is explained as the implementation of the principles of sustainable development in the built environment.

Kheni and Akoogo (2015) investigated the trend of sustainable construction in the built environment, which started with a decrease in energy use in order to reduce the environmental impact of construction processes. Furthermore, quality of building materials, components, elements and the type of technology involved were also considered. In addition, sustainable designs were also implemented. Currently, the issue of sustainability in the built environment has gone beyond just the environmental sustainability, as economic and social sustainability have also been incorporated. There is new thinking that requires individuals, construction companies and societies to find models, standards and tools to clarify the extent and ways, in which current activities are not sustainable, and develop a standard scale that will contribute to achieving sustainability in all its forms.

Dania et al. (2013) conducted a study on mainstreaming sustainable construction for indigenous and multinational companies in Nigeria. The study concluded that multinational companies had a higher capacity, organisation, higher awareness level, and knowledge base for sustainable construction practices than the indigenous companies. However, it only confirmed the ability of companies to implement sustainability strategies but was unable to establish the relationship between the adoption of sustainable construction practices and firm performance.

Waziri, Yuso, and Osmadi (2015) studied the implementation of sustainable building practices in Nigeria. They stated that a sustainable practice is lightly implemented at the firm level, while moderately implemented at the individual and project levels. However, they were unable to evaluate the factors that accounted for poor implementation, nor did they determine the impact of low adoption rates on business performance. Other studies conducted on sustainability issues in Nigeria include Ikediashi et al. (2012), Ujene (2014), Ijaiya (2014), Ekung et al. (2014), Barde and Tela (2015) and Ujene and Oladokun (2017), with a very scarce study in the Niger Delta. These studies did not establish the influence of the adoption level of sustainable construction practices on firm performance.

Combs, Crook, and Shook, (2005) revealed that firm performance suffered from a problem of incompatibility and an appropriate consideration for its dimension. Many researchers often use the financial performance measures and ignore the significance of environmental and social performance measures. Researchers present company performance as one-dimensional aspect despite it has been recognised to incorporate multi-dimensionality issues (Glick, Washburn, and Miller, 2005). Shen et al. (2010) stated that the incorporation of economic and social performance parameters into firm performance measurement was gaining popularity among scholars. However, Tam (2008) opined that sustainability in the built environment was often viewed in relation to the environment. Given this 
problem, this study examined the sustainability practices of construction companies in Niger-Delta, (Nigeria) and their contribution to corporate performance.

Critical theory and stakeholder theory are theories that support the model in the present research. The critical theory was applied in the study because of the need to determine the extent of implementation of sustainable construction practices among construction companies and to create an enhanced set of corporate knowledge of sustainability. This, in turn, will reduce the level of conflict and tension between the companies and people of the Niger Delta. The theoretical background used to visualize company performance in the study was stakeholder theory (Agle, Mitchell, and Sonnenfeld, 1999; Kaplan \& Norton, 1992; Waddock \& Graves, 1997).

Some studies measured performance indicators, but a majority of the measurements were project-based. There are few studies that assessed performance at the corporate level (Ali, Al-Sulaihi, and Al-Gahtani, 2012). In view of this fact, this study assessed a set of KPIs that can be applied by construction managers in measuring construction performance at the corporate level in the Niger Delta and Nigeria at large. Variables for measuring the level of sustainability adoption are firm sustainability practices, and there are ten of them in this study. These include: leadership (Inkoom, 2013), knowledge management practices (Sommerville \& Craig, 2006), organisational innovativeness (Widen, 2003), organisational culture (D’Incognito, Costantino \& Migliaccio, 2013; Al-Jamea, 2014), corporate governance (Eccles, Ioannun, and Serafeim, 2012), stakeholder engagement (Freeman, Harrison, and Wicks, 2007), transparency and measurement (Epstein, 2008), corporate social responsibility (Crowther, 2000), employment practices (Epstein, 2008) and environmental protection (Inkoom, 2013).

Several studies supported the notion that there was a positive relationship between sustainable building practices and company performance (Uadiale \& Fegbemi, 2012; Weshah et al., 2012; Waddock \& Graves, 1997). However, some argued that the implementation of sustainable construction practices negatively influenced firm performance. In view of this information, the aim of this study was to establish the relationship between the level of implementation of sustainable construction practices and performance of construction firms using structural equation modelling approach.

\section{RESEARCH METHODOLOGY}

In tandem with the survey approach adopted for the study, copies of structured questionnaire were administered to the representatives of the firms: project managers, architects, builders and civil engineers. A five-point (1-5) Likert scale was used for data collection (Kazaz, Manisali, and Ulubeyli, 2008; Santos \& Brito, 2012). Scale 1 signifies a very low level of implementation/very low level of performance, while scale 5 signifies a very high level of implementation/very high level of performance for the level of implementation of sustainable construction practices and firm performance, respectively (Kazaz, Manisali, and Ulubeyli, 2008; Santos \& Brito, 2012). Data were analysed using descriptive statistics and structural equation modelling. Structural equation modelling was used to establish the 
relationship between the adoption level of sustainable construction practices and firm performance in Niger Delta, Nigeria.

\subsection{Sample Frame and Sample Size}

Figure 1 shows that Bayelsa has the least number of construction firms operating in the study area, while River State has the highest number of construction companies operating in its surrounding area. This can be attributed to the volume of economic and construction activities taking place in the states. The sample frame and sample size are 1781 and 1179 , respectively.

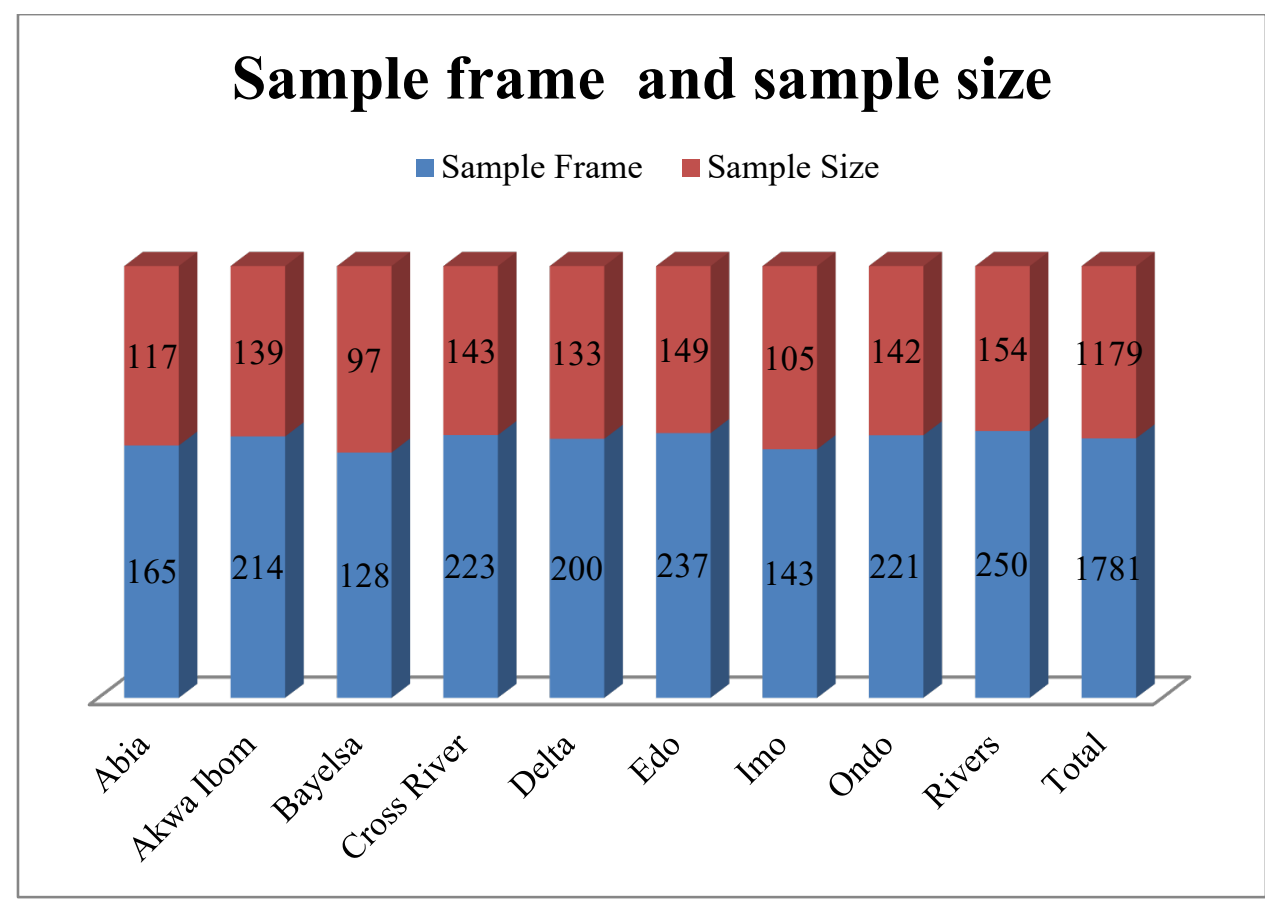

Fig. 1. Sample frame and sample size (developed by the authors).

This research applied Yamane (1967) equation for computation of the sample size as shown in Fig. 1. Equation for sample size is provided in Eq. (1):

where

$$
n=N / 1+N(e)^{2} \text {, }
$$

$n=$ sample size;

$N=$ finite population;

$e=$ level of significance $(0.05)$;

1 unity.

\subsection{Questionnaire Administration and Response Rate in the Study}

Structured questionnaire was used for data collection in the study.

Figure 2 shows that the response rate ranged between $76.10 \%$ and $90.10 \%$. It shows that a good number of the questionnaires was returned. 


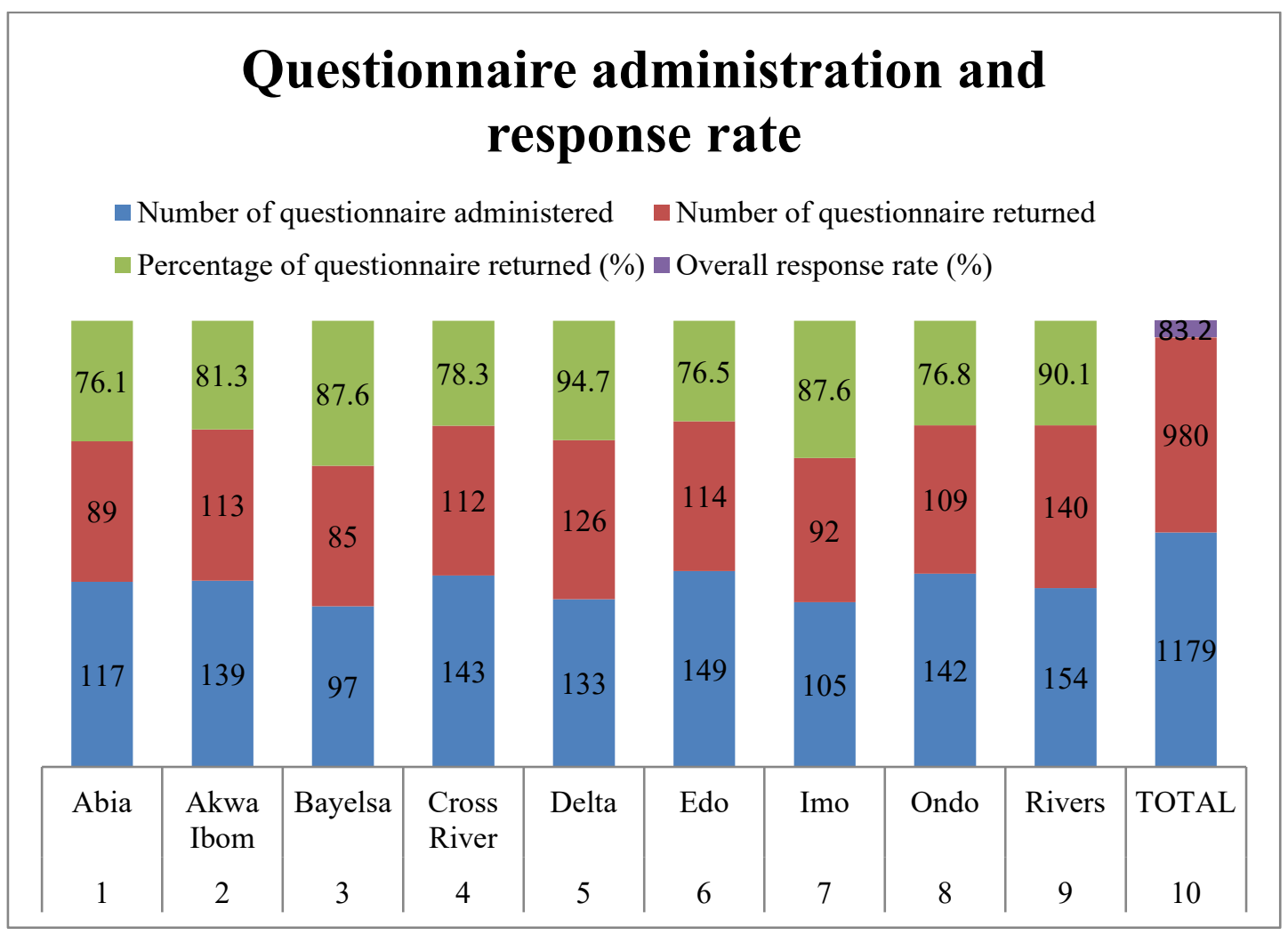

Fig. 2. Questionnaire distribution and response rate (developed by the authors).

It also indicates that firms operating in River State had the highest response rate $(90.10 \%)$, while the construction firms operating in Abia State had the lowest response rate $(76.10 \%)$.

\section{RESULTS OF THE RESEARCH}

\subsection{Structural Equation Modelling (SEM) for Predicting Performance of Construction Firms from the Adoption of Sustainable Construction Practices}

Structural equation modelling was used to predict performance of construction firms from the adoption of sustainable building practices in Niger Delta, Nigeria. These processes include model specification, model estimation and modification, verification and validation of the final model.

\section{Specification of SEM}

In SEM specification, the researchers justify the theories underpinning the relationship among the constructs and variables in literature. The model is based on critical and stakeholder theory. Causal relationship between the variables helped to gain a better understanding of the variable exploratory power. 




Fig. 3. Hypothesized structural equation model for predicting the performance of construction firms from the adoption of sustainability practices (developed by the authors). 
Figure 3 shows the initial hypothetical model for predicting the performance of construction firms from implementation of sustainable building practices in Niger Delta, Nigeria. Model fit tests were carried out on the initial model and the results are presented in Table 1. The results showed that the initial model did not satisfy the model fit indices as specified in literature. Hence, there is a need for the final model as indicated in Fig. 4.

\section{Model Estimation and Modification}

Maximum likelihood estimate (MLE) was applied in the study to determine the unique contrast among the elements. The Promax rotation method was used to reveal the underlying dimensions that formed contrast patterns among the variables. Moreover, the Promax rotation method was used because of the large data involved, and it also accounted for the correlation among variables.

The result showed that KMO was 0.941 , which confirmed the appropriateness and adequacy for conducting a factor analysis for the data set. This value is greater than the cut off of 0.5 for KMO and Bartlett's test $<0.05$. This confirmed the appropriateness and adequacy for conducting the factor analysis for the data set.

The study was not about grouping data because the breakdown of sustainability practices into categories from the literature was made at a preliminary stage. In the same way, sustainability practices, constructs, and variables beneath each construct and firm performance indicators were already categorized at the preliminary phase. PCA was used in this study to reduce the variables and find the smallest components that explained most of the variations in the data set. The result of the rotated matrix of components indicates that no component had less than three variables. It was also found that the correlation $r$ is greater than 0.5 for each variable. This shows a strong relationship among the variables. This also met the condition stated in the literature by Tabachnick and Fidell (2007), who argued that the correlation $r$ had to be 0.30 or greater, because anything below suggested a really weak relationship among the variables.

\section{Verification and Validation of the Final Model}

The final model was validated using the model fit indices. The results in Table 1 show that the model fit indices of the final model satisfied the bench marks set in literature. The results show that the estimates are within the expected limits. The model parameters indicated in Table 2 Tables 2-6 demonstrate that the final model is acceptable because estimates are within the stipulated bench marks in literature.

The standard error (S.E) shown in Table 2 Tables2-6 revealed a high level of accuracy of parameter estimation. The standard errors in this study were neither too high nor too small. They fell within the accepted range, which proved a strong model. The results show that the $p$-values are less than 0.05 . This implies that the estimates are significant at $95 \%$ confidence level. The values of chi-square, goodness of fit, root mean square, error of approximation, comparative fit index, Tucker-Lewis index, normed fit index, incremental fit index and relative index are $1.972,0.945,0.064,0.962,0.956,0.938,0.942$ and 0.941 , respectively. The values of these model fit indices showed a satisfactory model fit. This study is in tandem 
with Schumacher and Lomax (2004), Zulu (2007), Hair et al. (2010) and Byrne (2010) that used many model fit indices to determine the overall fitness of the model.

Table 1. Model Fit Indices (developed by the authors)

\begin{tabular}{|c|c|c|c|c|c|}
\hline Model Fit Indices & Recommended & Source(s) & $\begin{array}{l}\text { Initial } \\
\text { Model }\end{array}$ & $\begin{array}{l}\text { Final } \\
\text { Model }\end{array}$ & Remark \\
\hline $\begin{array}{l}\mathrm{X}^{2} / \text { Degree of } \\
\text { Freedom }\end{array}$ & $<2$ & Byrne, 2001 & 8.50 & 1.972 & satisfactory \\
\hline $\begin{array}{l}\text { Goodness of Fit } \\
\text { (GFI) }\end{array}$ & $0-1$ & $\begin{array}{l}\text { Bagozzi \& Yi, } \\
2012\end{array}$ & 0.723 & 0.945 & satisfactory \\
\hline $\begin{array}{l}\text { Root Mean Square } \\
\text { Error of } \\
\text { Approximation } \\
\text { (RMSEA) }\end{array}$ & $\leq 0.10$ & $\begin{array}{l}\text { Tabachnick \& } \\
\text { Fidell, } 2007\end{array}$ & 0.126 & 0.064 & satisfactory \\
\hline $\begin{array}{l}\text { Comparative Fit } \\
\text { Index (CFI) }\end{array}$ & $>0.9$ & Kline, 2005 & 0.755 & 0.962 & satisfactory \\
\hline $\begin{array}{l}\text { Tucker-Lewis } \\
\text { Index (TLI) }\end{array}$ & $\geq 0.90$ & $\begin{array}{l}\text { Bagozzi \& Yi, } \\
2012\end{array}$ & 0.884 & 0.956 & satisfactory \\
\hline $\begin{array}{l}\text { Normed Fit Index } \\
\text { (NFI) }\end{array}$ & $0-1$ & $\begin{array}{l}\text { Doloi, Iyer \& } \\
\text { Sawhney, } 2011\end{array}$ & 0.887 & 0.938 & satisfactory \\
\hline $\begin{array}{l}\text { Incremental Fit } \\
\text { Index (IFI) }\end{array}$ & $0-1$ & $\begin{array}{l}\text { Molenaar, } \\
\text { Washington \& } \\
\text { Diekmann, 2000 }\end{array}$ & 0.866 & 0.942 & satisfactory \\
\hline $\begin{array}{l}\text { Relative Fit Index } \\
\text { (RFI) }\end{array}$ & $0-1$ & $\begin{array}{l}\text { Doloi, Iyer \& } \\
\text { Sawhney, } 2011\end{array}$ & 0.856 & 0.941 & satisfactory \\
\hline
\end{tabular}

Table 2 shows the estimates of the standardised regression weight, the standard errors, and the $p$-values. Furthermore, another set of criteria to assess the adequacy of the modelist he feasibility of parameter estimates, the appropriateness of standard errors, and the statistical significance of parameter estimates. To check the feasibility, the parameters were checked to ensure that the estimates were correct. The correctness of the estimates implies that each of estimates has the correct values and sign. This is to avoid estimates having the correlation coefficient greater than one (1). The appropriateness of the standard error is also a key parameter for evaluating the adequacy of the model. Table 2 indicates that the values of standard errors are small, which implies a good model fit. The results in Table 2 also show that all the $p$-values are less than 0.05 level of significant set for the test. This indicates that the estimates are significant at $95 \%$ confidence level. These criteria and checks show that the model fit is good.

Table 2. Regression Weights: Group Number 1 - Default Model (developed by the authors)

\begin{tabular}{|l|l|l|r|r|l|}
\hline & & & Estimate & S.E. & $\begin{array}{l}\boldsymbol{P} \text { - } \\
\text { value }\end{array}$ \\
\hline $\begin{array}{l}\text { FIRMPERFORMA } \\
\text { NCE }\end{array}$ & $\begin{array}{l}<-- \\
-\end{array}$ & $\begin{array}{l}\text { ADOPTIONOFSUSTAINABILT } \\
\text { Y_PRACTICES }\end{array}$ & 0.923 & & \\
\hline CLIIAD & $\begin{array}{l}<-- \\
-\end{array}$ & $\begin{array}{l}\text { ADOPTIONOFSUSTAINABILT } \\
\text { Y_PRACTICES }\end{array}$ & 0.855 & & \\
\hline
\end{tabular}




\begin{tabular}{|c|c|c|c|c|c|}
\hline & & & Estimate & S.E. & $\begin{array}{l}P \text { - } \\
\text { value }\end{array}$ \\
\hline CLIMAD & $\begin{array}{l}<-- \\
-\end{array}$ & $\begin{array}{l}\text { ADOPTIONOFSUSTAINABILT } \\
\text { Y_PRACTICES }\end{array}$ & 0.831 & & \\
\hline ISAD & $\begin{array}{ll}<- \\
-\end{array}$ & $\begin{array}{l}\text { ADOPTIONOFSUSTAINABILT } \\
\text { Y_PRACTICES }\end{array}$ & 0.823 & & \\
\hline ICAD & $\begin{array}{l}<-- \\
- \\
\end{array}$ & $\begin{array}{l}\text { ADOPTIONOFSUSTAINABILT } \\
\text { Y_PRACTICES }\end{array}$ & 0.833 & & \\
\hline TMAD & $\begin{array}{l}<- \\
-\end{array}$ & $\begin{array}{l}\text { ADOPTIONOFSUSTAINABILT } \\
\text { Y_PRACTICES }\end{array}$ & 0.675 & & \\
\hline CGSGAD & $\begin{array}{ll}<-- \\
-\end{array}$ & $\begin{array}{l}\text { ADOPTIONOFSUSTAINABILT } \\
\text { Y_PRACTICES }\end{array}$ & 0.592 & & \\
\hline CSRAD & $\begin{array}{l}<-- \\
-\end{array}$ & $\begin{array}{l}\text { ADOPTIONOFSUSTAINABILT } \\
\text { Y_PRACTICES }\end{array}$ & 0.624 & & \\
\hline CGSRAD & $\begin{array}{ll}<- \\
-\end{array}$ & $\begin{array}{l}\text { ADOPTIONOFSUSTAINABILT } \\
\text { Y_PRACTICES }\end{array}$ & 0.568 & & \\
\hline OCPAD & $\begin{array}{ll}<-- \\
-\end{array}$ & $\begin{array}{l}\text { ADOPTIONOFSUSTAINABILT } \\
\text { Y_PRACTICES }\end{array}$ & 0.659 & & \\
\hline KMPAD & $\begin{array}{l}<-- \\
-\end{array}$ & $\begin{array}{l}\text { ADOPTIONOFSUSTAINABILT } \\
\text { Y_PRACTICES }\end{array}$ & 0.630 & & \\
\hline PEAD & $\begin{array}{l}<-- \\
-\end{array}$ & $\begin{array}{l}\text { ADOPTIONOFSUSTAINABILT } \\
\text { Y_PRACTICES }\end{array}$ & 0.634 & & \\
\hline OIESAD & $\begin{array}{ll}<- \\
- \\
\end{array}$ & $\begin{array}{l}\text { ADOPTIONOFSUSTAINABILT } \\
\text { Y_PRACTICES }\end{array}$ & 0.664 & & \\
\hline AEG & $\begin{array}{l}<-- \\
-\end{array}$ & FIRMPERFORMANCE & 0.600 & & \\
\hline NI & $\begin{array}{l}<- \\
-\end{array}$ & FIRMPERFORMANCE & 0.574 & & \\
\hline GES & $\begin{array}{l}<-- \\
-\end{array}$ & FIRMPERFORMANCE & 0.719 & & \\
\hline SP & $\begin{array}{ll}<-- \\
-\end{array}$ & FIRMPERFORMANCE & 0.811 & & \\
\hline RBOC & $\begin{array}{l}<-- \\
-\end{array}$ & FIRMPERFORMANCE & 0.660 & & \\
\hline AFT & $\begin{array}{l}<-- \\
-\end{array}$ & FIRMPERFORMANCE & 0.615 & & \\
\hline CLII6AD & $\begin{array}{ll}<-- \\
-\end{array}$ & CLIIAD & 0.595 & & \\
\hline CLII5AD & $\begin{array}{l}<-- \\
-\end{array}$ & CLIIAD & 0.840 & 0.053 & $* * *$ \\
\hline CLII4AD & $\begin{array}{l}<-- \\
-\end{array}$ & CLIIAD & 0.807 & 0.056 & $* * *$ \\
\hline CLII3AD & $\begin{array}{l}<-- \\
-\end{array}$ & CLIIAD & 0.745 & 0.056 & $* * *$ \\
\hline CLII2AD & $\begin{array}{l}<-- \\
-\end{array}$ & CLIIAD & 0.849 & 0.056 & $* * *$ \\
\hline CLII1AD & $\begin{array}{ll}<-- \\
-\end{array}$ & CLIIAD & 0.773 & 0.056 & $* * *$ \\
\hline TM10AD & $\begin{array}{l}<-- \\
-\end{array}$ & TMAD & 0.647 & & \\
\hline
\end{tabular}




\begin{tabular}{|c|c|c|c|c|c|}
\hline & & & Estimate & S.E. & $\begin{array}{l}P \text { - } \\
\text { value }\end{array}$ \\
\hline TM8AD & $\begin{array}{l}<-- \\
-\end{array}$ & TMAD & 0.778 & 0.052 & $* * *$ \\
\hline TM5AD & $\begin{array}{l}<-- \\
-\end{array}$ & TMAD & 0.805 & 0.048 & $* * *$ \\
\hline TM4AD & $\begin{array}{l}<-- \\
-\end{array}$ & TMAD & 0.918 & 0.045 & $* * *$ \\
\hline TM3AD & \begin{tabular}{|l|}
$<-$ \\
- \\
\end{tabular} & TMAD & 0.851 & 0.046 & $* * *$ \\
\hline TM2AD & \begin{tabular}{|l|}
$<-$ \\
- \\
\end{tabular} & TMAD & 0.797 & 0.049 & $* * *$ \\
\hline CLIM6AD & \begin{tabular}{|l|}
$<--$ \\
- \\
\end{tabular} & CLIMAD & 0.673 & & \\
\hline CLIM5AD & \begin{tabular}{|l|}
$<--$ \\
- \\
\end{tabular} & CLIMAD & 0.768 & 0.055 & $* * *$ \\
\hline CLIM4AD & \begin{tabular}{|l|}
$<--$ \\
-
\end{tabular} & CLIMAD & 0.864 & 0.051 & $* * *$ \\
\hline CLIM3AD & \begin{tabular}{l|}
$<--$ \\
- \\
\end{tabular} & CLIMAD & 0.861 & 0.053 & $* * *$ \\
\hline CLIM2AD & \begin{tabular}{|l|}
$<--$ \\
- \\
\end{tabular} & CLIMAD & 0.829 & 0.053 & $* * *$ \\
\hline CLIM1 AD & \begin{tabular}{|l|}
$<--$ \\
- \\
\end{tabular} & CLIMAD & 0.844 & 0.050 & $* * *$ \\
\hline KMP2AD & \begin{tabular}{|l|}
$<--$ \\
- \\
\end{tabular} & KMPAD & 0.704 & & \\
\hline KMP4AD & $\begin{array}{l}<-- \\
- \\
\end{array}$ & KMPAD & 0.929 & 0.055 & $* * *$ \\
\hline KMP5AD & $\begin{array}{l}<-- \\
- \\
\end{array}$ & KMPAD & 0.920 & 0.049 & $* * *$ \\
\hline KMP6AD & $\begin{array}{l}<-- \\
- \\
\end{array}$ & KMPAD & 0.917 & 0.047 & $* * *$ \\
\hline KMP7AD & \begin{tabular}{|l|}
$<-$ \\
- \\
\end{tabular} & KMPAD & 0.680 & 0.043 & $* * *$ \\
\hline IC5AD & \begin{tabular}{|l|}
$<--$ \\
- \\
\end{tabular} & ICAD & 0.698 & & \\
\hline IC4AD & \begin{tabular}{|l|}
$<--$ \\
- \\
\end{tabular} & ICAD & 0.768 & 0.055 & $* * *$ \\
\hline IC3AD & \begin{tabular}{|l|}
$<--$ \\
- \\
\end{tabular} & ICAD & 0.873 & 0.059 & $* * *$ \\
\hline IC $2 A D$ & \begin{tabular}{|l|}
$<--$ \\
- \\
\end{tabular} & ICAD & 0.718 & 0.047 & $* * *$ \\
\hline IC1AD & \begin{tabular}{|l|}
$<--$ \\
-
\end{tabular} & ICAD & 0.675 & 0.052 & $* * *$ \\
\hline CGSG1AD & \begin{tabular}{|l|}
$<--$ \\
-
\end{tabular} & CGSGAD & 0.806 & & \\
\hline CGSG2AD & \begin{tabular}{|l|}
$<-$ \\
- \\
\end{tabular} & CGSGAD & 0.921 & 0.046 & $* * *$ \\
\hline CGSG4AD & \begin{tabular}{|l|}
$<-$ \\
-
\end{tabular} & CGSGAD & 0.707 & 0.037 & $* * *$ \\
\hline
\end{tabular}




\begin{tabular}{|c|c|c|c|c|c|}
\hline & & & Estimate & S.E. & $\begin{array}{l}P \text { - } \\
\text { value }\end{array}$ \\
\hline GES3 & $\begin{array}{l}<-- \\
-\end{array}$ & GES & 0.807 & & \\
\hline GES5 & $\begin{array}{l}<-- \\
-\end{array}$ & GES & 0.852 & 0.038 & $* * *$ \\
\hline GES6 & $\begin{array}{l}<-- \\
-\end{array}$ & GES & 0.737 & 0.045 & $* * *$ \\
\hline GES7 & \begin{tabular}{|l|}
$<--$ \\
- \\
\end{tabular} & GES & 0.864 & 0.041 & $* * *$ \\
\hline CGSR2AD & \begin{tabular}{|l|}
$<-$ \\
- \\
\end{tabular} & CGSRAD & 0.679 & & \\
\hline CGSR3AD & \begin{tabular}{|l|}
$<--$ \\
-
\end{tabular} & CGSRAD & 0.842 & 0.059 & $* * *$ \\
\hline CGSR4AD & \begin{tabular}{l|}
$<--$ \\
- \\
\end{tabular} & CGSRAD & 0.582 & 0.085 & $* * *$ \\
\hline CGSR6AD & \begin{tabular}{|l|}
$<--$ \\
-
\end{tabular} & CGSRAD & 0.864 & 0.056 & $* * *$ \\
\hline CGSR7AD & \begin{tabular}{l|}
$<--$ \\
- \\
\end{tabular} & CGSRAD & 0.856 & 0.052 & $* * *$ \\
\hline CGSR8AD & \begin{tabular}{|l|}
$<--$ \\
- \\
\end{tabular} & CGSRAD & 0.867 & 0.052 & $* * *$ \\
\hline CGSR9AD & \begin{tabular}{|l|}
$<--$ \\
- \\
\end{tabular} & CGSRAD & 0.834 & 0.044 & $* * *$ \\
\hline CGSR10AD & \begin{tabular}{|l|}
$<--$ \\
- \\
\end{tabular} & CGSRAD & 0.512 & 0.083 & $* * *$ \\
\hline CGSR11AD & \begin{tabular}{|l|}
$<--$ \\
- \\
\end{tabular} & CGSRAD & 0.867 & 0.044 & $* * *$ \\
\hline CGSR12AD & $\begin{array}{l}<-- \\
- \\
\end{array}$ & CGSRAD & 0.903 & 0.043 & $* * *$ \\
\hline CGSR13AD & $\begin{array}{l}<-- \\
- \\
\end{array}$ & CGSRAD & 0.864 & 0.046 & $* * *$ \\
\hline CGSR14AD & \begin{tabular}{|l|}
$<-$ \\
- \\
\end{tabular} & CGSRAD & 0.853 & 0.040 & $* * *$ \\
\hline CGSR15AD & \begin{tabular}{|l|}
$<--$ \\
- \\
\end{tabular} & CGSRAD & 0.848 & 0.043 & $* * *$ \\
\hline CGSR16AD & \begin{tabular}{|l|}
$<--$ \\
- \\
\end{tabular} & CGSRAD & 0.851 & 0.039 & $* * *$ \\
\hline AEG1 & \begin{tabular}{|l|}
$<--$ \\
- \\
\end{tabular} & AEG & 0.801 & & \\
\hline AEG2 & \begin{tabular}{|l|}
$<--$ \\
-
\end{tabular} & AEG & 0.957 & 0.034 & $* * *$ \\
\hline AEG3 & \begin{tabular}{|l|}
$<--$ \\
-
\end{tabular} & AEG & 0.978 & 0.035 & $* * *$ \\
\hline AEG4 & \begin{tabular}{|l|}
$<--$ \\
-
\end{tabular} & AEG & 0.857 & 0.036 & $* * *$ \\
\hline OIES2AD & \begin{tabular}{|l|}
$<-$ \\
- \\
\end{tabular} & OIESAD & 0.780 & & \\
\hline OIES3AD & \begin{tabular}{|l|}
$<-$ \\
-
\end{tabular} & OIESAD & 0.702 & 0.047 & $* * *$ \\
\hline
\end{tabular}




\begin{tabular}{|c|c|c|c|c|c|}
\hline & & & Estimate & S.E. & $\begin{array}{l}P \text { - } \\
\text { value }\end{array}$ \\
\hline OIES4AD & $\begin{array}{l}<-- \\
-\end{array}$ & OIESAD & 0.618 & 0.050 & $* * *$ \\
\hline IS6AD & $\begin{array}{l}<-- \\
-\end{array}$ & ISAD & 0.651 & & \\
\hline IS5AD & $\begin{array}{l}<-- \\
-\end{array}$ & ISAD & 0.835 & 0.052 & $* * *$ \\
\hline IS4AD & \begin{tabular}{|l|}
$<--$ \\
- \\
\end{tabular} & ISAD & 0.790 & 0.056 & $* * *$ \\
\hline IS3AD & \begin{tabular}{|l|}
$<--$ \\
-
\end{tabular} & ISAD & 0.732 & 0.058 & $* * *$ \\
\hline IS2AD & \begin{tabular}{|l|}
$<--$ \\
- \\
\end{tabular} & ISAD & 0.800 & 0.057 & $* * *$ \\
\hline IS1AD & \begin{tabular}{l|}
$<--$ \\
- \\
\end{tabular} & ISAD & 0.762 & 0.054 & $* * *$ \\
\hline PE4AD & \begin{tabular}{|l|}
$<--$ \\
-
\end{tabular} & PEAD & 0.841 & & \\
\hline PE5AD & \begin{tabular}{|l|}
$<--$ \\
-
\end{tabular} & PEAD & 0.863 & 0.035 & $* * *$ \\
\hline PE6AD & \begin{tabular}{|l|}
$<--$ \\
- \\
\end{tabular} & PEAD & 0.874 & 0.037 & $* * *$ \\
\hline PE7AD & \begin{tabular}{|l|}
$<--$ \\
- \\
\end{tabular} & PEAD & 0.857 & 0.035 & $* * *$ \\
\hline PE8AD & \begin{tabular}{|l|}
$<--$ \\
- \\
\end{tabular} & PEAD & 0.844 & 0.035 & $* * *$ \\
\hline PE9AD & $\begin{array}{l}<-- \\
- \\
\end{array}$ & PEAD & 0.837 & 0.038 & $* * *$ \\
\hline PE10AD & $\begin{array}{l}<-- \\
- \\
\end{array}$ & PEAD & 0.908 & 0.033 & $* * *$ \\
\hline PE11AD & $\begin{array}{l}<-- \\
- \\
\end{array}$ & PEAD & 0.826 & 0.037 & $* * *$ \\
\hline CSR4AD & \begin{tabular}{|l|}
$<-$ \\
- \\
\end{tabular} & CSRAD & 0.818 & & \\
\hline CSR5AD & \begin{tabular}{|l|}
$<--$ \\
- \\
\end{tabular} & CSRAD & 0.877 & 0.036 & $* * *$ \\
\hline CSR6AD & \begin{tabular}{|l|}
$<--$ \\
- \\
\end{tabular} & CSRAD & 0.894 & 0.033 & $* * *$ \\
\hline CSR10AD & \begin{tabular}{|l|}
$<--$ \\
- \\
\end{tabular} & CSRAD & 0.596 & 0.048 & $* * *$ \\
\hline NI1 & \begin{tabular}{|l|}
$<--$ \\
-
\end{tabular} & NI & 0.992 & & \\
\hline NI2 & \begin{tabular}{|l|}
$<--$ \\
-
\end{tabular} & NI & 0.987 & 0.009 & $* * *$ \\
\hline NI3 & \begin{tabular}{|l|}
$<--$ \\
-
\end{tabular} & NI & 0.923 & 0.016 & $* * *$ \\
\hline NI4 & \begin{tabular}{|l|}
$<-$ \\
- \\
\end{tabular} & NI & 0.412 & 0.050 & $* * *$ \\
\hline NI5 & \begin{tabular}{|l|}
$<-$ \\
-
\end{tabular} & NI & 0.660 & 0.028 & $* * *$ \\
\hline
\end{tabular}




\begin{tabular}{|c|c|c|c|c|c|}
\hline & & & Estimate & S.E. & $\begin{array}{l}P \text { - } \\
\text { value }\end{array}$ \\
\hline NI6 & $\begin{array}{l}<-- \\
-\end{array}$ & $\mathrm{NI}$ & 0.534 & 0.028 & $* * *$ \\
\hline SP3 & $\begin{array}{l}<-- \\
-\end{array}$ & SP & 0.812 & & \\
\hline SP4 & $\begin{array}{l}<-- \\
-\end{array}$ & SP & 0.833 & 0.039 & $* * *$ \\
\hline SP5 & \begin{tabular}{|l|}
$<--$ \\
-
\end{tabular} & SP & 0.893 & 0.041 & $* * *$ \\
\hline SP6 & \begin{tabular}{|l|}
$<--$ \\
- \\
\end{tabular} & SP & 0.906 & 0.040 & $* * *$ \\
\hline SP7 & \begin{tabular}{l|}
$<--$ \\
-
\end{tabular} & SP & 0.894 & 0.038 & $* * *$ \\
\hline SP8 & $\begin{array}{l}<-- \\
-\end{array}$ & SP & 0.887 & 0.039 & $* * *$ \\
\hline SP9 & $\begin{array}{l}<-- \\
-\end{array}$ & SP & 0.875 & 0.045 & *** \\
\hline RBOC5 & \begin{tabular}{|l|}
$<--$ \\
-
\end{tabular} & RBOC & 0.822 & & \\
\hline RBOC6 & \begin{tabular}{|l|}
$<--$ \\
- \\
\end{tabular} & RBOC & 0.892 & 0.038 & $* * *$ \\
\hline RBOC7 & \begin{tabular}{|l|}
$<--$ \\
- \\
\end{tabular} & $\mathrm{RBOC}$ & 0.826 & 0.037 & $* * *$ \\
\hline RBOC8 & \begin{tabular}{|l|}
$<--$ \\
-
\end{tabular} & RBOC & 0.633 & 0.034 & *** \\
\hline OCP2AD & \begin{tabular}{|l|}
$<--$ \\
- \\
\end{tabular} & OCPAD & 0.834 & 0.057 & $* * *$ \\
\hline OCP3AD & \begin{tabular}{|l|}
$<--$ \\
- \\
\end{tabular} & OCPAD & 0.866 & 0.059 & $* * *$ \\
\hline OCP4AD & $\begin{array}{l}<-- \\
-\end{array}$ & OCPAD & 0.727 & 0.056 & $* * *$ \\
\hline AFT3 & \begin{tabular}{l|}
$<--$ \\
- \\
\end{tabular} & AFT & 0.848 & & \\
\hline AFT4 & \begin{tabular}{|l|}
$<--$ \\
- \\
\end{tabular} & AFT & 0.971 & 0.029 & $* * *$ \\
\hline AFT5 & \begin{tabular}{|l|}
$<--$ \\
-
\end{tabular} & AFT & 0.890 & 0.029 & $* * *$ \\
\hline
\end{tabular}

S.E. - standard error

Table 3 shows the squared multiple correlations of each of the variables in the model. Table 3 reveals the proportion of variability in firm performance as explained by the level of adoption of sustainability practices. The implication of each of the estimates in Table 3 is that each variable is considered to be a dependent variable. For example, the construction firm performance with squared multiple correlation of 0.853 means that $85.3 \%$ of the variability in the performance of construction firms in Niger Delta is explained by the level of adoption of sustainability practices. 
Table 3. Squared Multiple Correlations: Group Number 1 - Default Model (developed by the authors)

\begin{tabular}{|c|c|}
\hline & Estimate \\
\hline ADOPTIONOFSUSTAINABILTY_PRACTICES & 0.000 \\
\hline FIRMPERFORMANCE & 0.853 \\
\hline AFT & 0.378 \\
\hline OCPAD & 0.434 \\
\hline RBOC & 0.435 \\
\hline SP & 0.658 \\
\hline NI & 0.329 \\
\hline CSRAD & 0.390 \\
\hline PEAD & 0.402 \\
\hline ISAD & 0.677 \\
\hline OIESAD & 0.441 \\
\hline AEG & 0.360 \\
\hline CGSRAD & 0.323 \\
\hline GES & 0.516 \\
\hline CGSGAD & 0.350 \\
\hline ICAD & 0.694 \\
\hline KMPAD & 0.397 \\
\hline CLIMAD & 0.691 \\
\hline TMAD & 0.456 \\
\hline CLIIAD & 0.732 \\
\hline AFT5 & 0.792 \\
\hline AFT4 & 0.943 \\
\hline AFT3 & 0.719 \\
\hline OCP4AD & 0.528 \\
\hline OCP3AD & 0.751 \\
\hline OCP2AD & 0.696 \\
\hline RBOC8 & 0.401 \\
\hline RBOC7 & 0.682 \\
\hline RBOC6 & 0.796 \\
\hline RBOC5 & 0.675 \\
\hline SP9 & 0.765 \\
\hline SP8 & 0.786 \\
\hline SP7 & 0.799 \\
\hline SP6 & 0.821 \\
\hline SP5 & 0.797 \\
\hline SP4 & 0.694 \\
\hline SP3 & 0.659 \\
\hline NI6 & 0.285 \\
\hline NI5 & 0.436 \\
\hline NI4 & 0.170 \\
\hline NI3 & 0.851 \\
\hline NI2 & 0.974 \\
\hline NI1 & 0.983 \\
\hline CSR10AD & 0.355 \\
\hline CSR6AD & 0.798 \\
\hline
\end{tabular}


Baltic Journal of Real Estate Economics and Construction Management

\begin{tabular}{|c|c|}
\hline & Estimate \\
\hline CSR5AD & 0.769 \\
\hline CSR4AD & 0.668 \\
\hline PE11AD & 0.682 \\
\hline PE10AD & 0.824 \\
\hline PE9AD & 0.701 \\
\hline PE8AD & 0.712 \\
\hline PE7AD & 0.735 \\
\hline PE6AD & 0.764 \\
\hline PE5AD & 0.744 \\
\hline PE4AD & 0.707 \\
\hline IS1AD & 0.580 \\
\hline IS2AD & 0.640 \\
\hline IS3AD & 0.536 \\
\hline IS4AD & 0.624 \\
\hline IS5AD & 0.697 \\
\hline IS6AD & 0.424 \\
\hline OIES4AD & 0.382 \\
\hline OIES3AD & 0.493 \\
\hline OIES2AD & 0.609 \\
\hline AEG4 & 0.735 \\
\hline AEG3 & 0.956 \\
\hline AEG2 & 0.916 \\
\hline AEG1 & 0.642 \\
\hline CGSR16AD & 0.724 \\
\hline CGSR15AD & 0.719 \\
\hline CGSR14AD & 0.727 \\
\hline CGSR13AD & 0.746 \\
\hline CGSR12AD & 0.815 \\
\hline CGSR11AD & 0.751 \\
\hline CGSR10AD & 0.262 \\
\hline CGSR9AD & 0.696 \\
\hline CGSR8AD & 0.751 \\
\hline CGSR7AD & 0.733 \\
\hline CGSR6AD & 0.746 \\
\hline CGSR4AD & 0.339 \\
\hline CGSR3AD & 0.708 \\
\hline CGSR2AD & 0.461 \\
\hline GES7 & 0.747 \\
\hline GES6 & 0.544 \\
\hline GES5 & 0.726 \\
\hline GES3 & 0.651 \\
\hline CGSG4AD & 0.500 \\
\hline CGSG2AD & 0.849 \\
\hline CGSG1AD & 0.650 \\
\hline IC $1 A D$ & 0.456 \\
\hline IC2AD & 0.516 \\
\hline IC3AD & 0.762 \\
\hline IC4AD & 0.590 \\
\hline
\end{tabular}




\begin{tabular}{lr}
\hline & Estimate \\
\hline IC5AD & 0.488 \\
KMP7AD & 0.462 \\
KMP6AD & 0.840 \\
KMP5AD & 0.845 \\
KMP4AD & 0.863 \\
KMP2AD & 0.495 \\
CLIM1AD & 0.712 \\
CLIM2AD & 0.687 \\
CLIM3AD & 0.742 \\
CLIM4AD & 0.746 \\
CLIM5AD & 0.591 \\
CLIM6AD & 0.452 \\
TM2AD & 0.634 \\
TM3AD & 0.724 \\
TM4AD & 0.842 \\
TM5AD & 0.649 \\
TM8AD & 0.606 \\
TM10AD & 0.419 \\
CLII1AD & 0.597 \\
CLII2AD & 0.721 \\
CLII3AD & 0.555 \\
CLII4AD & 0.651 \\
CLII5AD & 0.706 \\
CLII6AD & 0.354 \\
\hline
\end{tabular}

The results show that the model fit is adequate and satisfactory.

\subsection{Relationship between the Adoption of Sustainable Construction Practices and Performance of Construction Firms}

Figure 4 reveals that the adoption level of sustainable construction practices has a direct and positive influence on the performance of construction firms in Niger Delta region of Nigeria $(\beta=0.923)$.

Table 3 shows the standardised direct effects of the variables. The result shows that $85.3 \%$ of the variability in the performance of construction firms in Niger Delta is explained by the adoption level of sustainable construction practices.

The annual employment growth with squared multiple correlation of 0.360 means that $36 \%$ of the variability in the annual employment growth is explained by the adoption level of sustainable construction practices. Also, the annual financial turnover with squared multiple correlation of 0.378 means that $37.8 \%$ of the variability in the annual financial turnover is explained by the adoption level of sustainable construction practices. In the same vein, the net income growth (profitability) of firms with squared multiple correlation of 0.329 means that $32.9 \%$ of the variability in net income is explained by the adoption level of sustainable construction practices. 


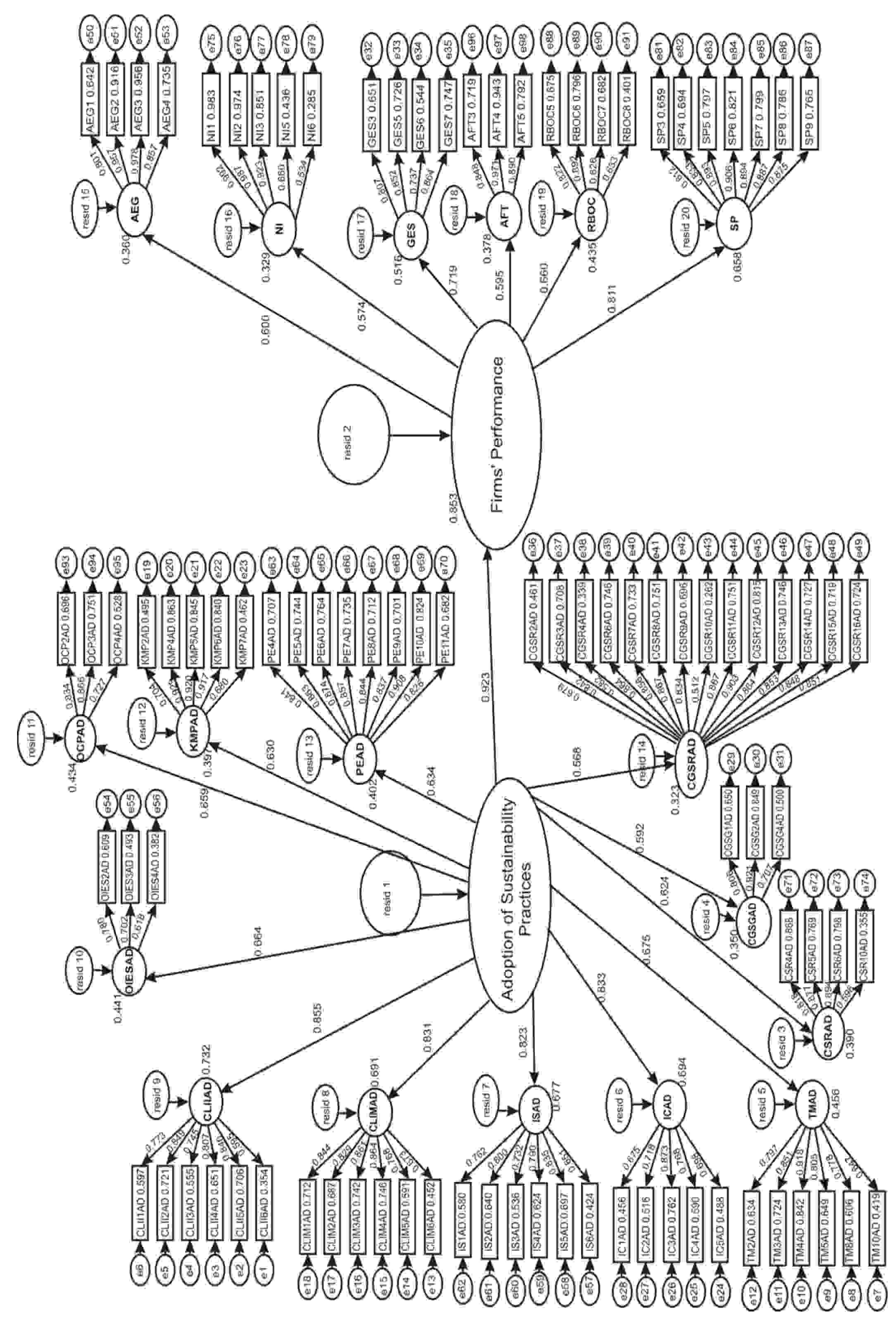

Fig. 4. Structural equation model predicting the performance of construction firms from the adoption of sustainability practices (developed by the authors). 
Furthermore, the remuneration and benefits with squared multiple correlation of 0.435 means that $43.5 \%$ of the variability in remuneration and benefits is explained by the adoption level of sustainable construction practices.

General employee satisfaction of firms with squared multiple correlation of 0.516 means that $51.6 \%$ of the variability in general employee satisfaction is explained by the adoption level of sustainable construction practices, and the social performance of firms with squared multiple correlation of 0.658 means that $65.8 \%$ of the variability in the social performance is explained by the adoption level of sustainable construction practices.

\section{DISCUSSION OF FINDINGS}

The $p$-value of 0.001 implies that there is a significant relationship between the adoption level of sustainable construction practices and the performance of construction firms. Correlation coefficient of 0.923 indicates that the adoption level of sustainable building practices has a direct and positive influence on construction firm performance. It was also revealed that $85.3 \%$ of the variability in the overall performance of construction firms was explained by the adoption level of sustainable construction practices. The study showed that $36 \%$ of the variability in the annual employment growth was explained by the adoption level of sustainable building practices. It was also demonstrated in the study that $37.8 \%$ of the variability in the annual financial turnover was explained by the level of adoption of sustainability practices. Also, $32.9 \%$ of the variability in net income was explained by the adoption level of sustainable building practices, while $43.5 \%$ of the variability in remuneration and benefits was explained by the adoption level of sustainable building practices. The study showed that $51.6 \%$ of the variability in general employee satisfaction was explained by the adoption level of sustainable building practices and $65.8 \%$ of the variability in the social performance was explained by the adoption level of sustainable building practices. This study is consistent with Maletič et al. (2014) who stated that sustainable and innovative practices influenced organisational performance.

\section{CONCLUSION}

This study investigated the sustainable construction practices and their contribution to firm performance. The study developed the structural equation model that predicted the performance of construction firm from the adoption level of sustainable construction practices. It was found out that there was a positive relationship between the implementation level of sustainable construction practices and firm performance. It was also concluded that the adoption level of sustainable construction practices accounted for $85 \%$ of the variability in the firm performance. It was also determined that the adoption level of sustainable construction practices accounted for $36 \%, 37.8 \%, 43.5 \%, 51.6 \%, 65.8 \%$, and $32.9 \%$ in the variability in annual employment growth, annual financial turnover, remuneration and benefits, general employee satisfaction, social performance and net income growth of the construction firms, respectively. This study concluded that proper 
implementation of sustainable construction practices at the firm level would lead to improvement in the firm performance. In tandem with the findings and conclusion of the study, the following recommendations are thus provided: The construction firms should not use only financial performance indicators to evaluate the level of their performance but rather apply a multi-dimensional approach, which includes financial performance indicators and non-financial performance indicators. This will enable the firms to have a holistic and broader knowledge of their performance. They should improve their adoption level of sustainable construction practices, which will lead to better or improved firm performance. The construction firms should adopt the structural equation model developed in this study to carry out integrated performance measurement.

\section{REFERENCES}

Agle, B. R., Mitchell, R. K., and Sonnenfeld, J. A. (1999). Who Matter to CEOs? An Investigation of Stakeholder Attributes and Salience Corporate Performance, and CEO values. Academy of Management Journal, 42(5), 507-525. https://doi.org/10.5465/256973

Ali, H. A. E. M, Al-Sulaihi, I. A., and Al-Gahtani, K. S. (2012). Indicators for Measuring Performance of Building Construction Companies in Kingdom of Saudi Arabia. Journal of King Saud University - Engineering Sciences, 25(2), 125-134. https://doi.org/10.1016/j.jksues.2012.03.002

Al-Jamea, M. (2014). Towards Social and Cultural Sustainability in the Designs of Contemporary Saudi Houses. Int. J. Sustain. Hum. Dev., 2(1), 35-43.

Bagozzi, R. P., and Yi, Y. (2012), Specification, evaluation, and interpretation of structural equation models. Journal of the Academy of Marketing Science, 40, 8-34. https://doi.org/10.1007/s11747$\underline{011-0278-\mathrm{X}}$

Barde, I. M., and Tela, G. (2015). Corporate Social Responsibility and Financial Performance in the Nigerian Construction Industry. International Conference on Humanities, Literature and Management (ICHLM'15), January 9-10, 2015 Dubai (UAE).

Brundtland, G. H. (1987). Our Common Future: Report of the World Commission in Environment and Development. Oxford: Oxford University Press.

Byrne, B.M. (2010). Structural Equation Modeling with AMOS: Basic Concepts, Applications and Programming, 3rd edition. Rutledge, Taylor and Francis, New York, NY.

Combs, J. G., Crook, T. R., and Shook, C. L. (2005). The Dimension of Organisational Performance and its Implications for Strategic Management Research. In D. J. Ketchen and D. D. Bergh (Eds.), Research Methodology in Strategy and Management, pp. 259-286. San Diego: Elsevier. https://doi.org/10.1016/S1479-8387(05)02011-4

Crowther, D. (2000). Social and Environmental Accounting. London: Financial Times Prentice Hall.

Dania, A. A, Larsen, G.D. and Yao, R. (2013). Mainstreaming Sustainable Construction: Case Studies of an Indigenous and Multinational Firm in Nigeria. Proceedings, Engineering Project Organization Conference Devil's Thumb Ranch, Colorado, USA.

D'Incognito, M., Costantino, N., and Migliaccio, G. C. (2013). Assessing the Influence of Cultural Issues on the Adoption of Life Cycle Management Tools. In S. Kajewski, K. Manley, and K. Hampson (Eds.), Proceedings of the 19th International CIB World Building Congress. Brisbane: Queensland University of Technology.

Doloi, H, Iyer, K. C., and Sawhney, A. (2011). Structural equation model for assessing impacts of contractor's performance on project success. International Journal of Project Management, 29, 687-695. https://doi.org/10.1016/j.ijproman.2010.05.007

Eccles, R. A., Ioannun, I., and Serafeim, G. (2012). The Impact of Corporate Sustainability on Organisational Processes and Performance, the National Bureau of Economic Research, Working Paper17950, NBER Working Paper Series. https://doi.org/10.3386/w17950 
Ekung, S. Ujene, A., \& Ebong, U. (2014). Drivers of Corporate Social Responsibility within Construction Organization in Nigeria. International Letters of Social and Humanistic Sciences, 32, 14-25. https://doi.org/10.18052/www.scipress.com/ILSHS.32.14

Elijidi-Ten, E. (2007). Applying Stakeholder Theory to Analyze Corporate Environmental Performance.Asian Review of Accounting, 15(2),164-184. https://doi.org/10.1108/13217340710823378

Emmitt, S., and Gorse, C. (2003). Construction Communication. Oxford: Blackwell Publishing.

Epstein, M. J. (2008). Making Sustainability Work: Best Practices in Managing and Measuring Corporate Social, Environmental and Economic Impacts. Berrett-Koehler Publishers.

Freeman, R. E., Harrison, J. S., and Wicks, A. C. (2007). Managing for Stakeholders: Survival, Reputation, and Success. New Haven, CT: Yale University Press, United States.

Friedman, M. (2007). The Social Responsibility of Business is to Increase its Profits. Springer, New York. https://doi.org/10.1007/978-3-540-70818-6_14

Glick, W. H., Washburn, N. T., and Miller, C. C. (2005). The Myth of Firm Performance. Proceedings of the Annual Meeting of American Academy of Management. Honolulu, Hawaii.

Groves, R. M. (2006). Nonresponse Rates and Nonresponse Bias in Household Surveys. Public Opinion Quarterly, 70(5), 646-675. https://doi.org/10.1093/poq $/ \mathrm{nfl} 033$

Hair, J. F., Anderson, R. E., Tatham, R. L., and Black, W. C. (1998). Multivariate data analysis: with readings. Prentice-Hall, Inc., Upper saddle river, NJ, USA.

Hair, J. F., Black, W. C., Babin, B. J., and Anderson, R. E. (2010). Multivariate data analysis: $a$ global perspective. Prentice-Hall, Inc., Englewood Cliffs, NJ, USA.

Ijaiya, H. (2014). Challenges of Corporate Social Responsibility in the Niger Delta Region of Nigeria, Afe Babalola University. Journal of Sustainable Development Law and Policy, 3(1), $60-71$.

Ijigah, E. A., Jimoh, R. A., Aruleba, B. O., and Ade, A. B. (2013). An Assessment of Environmental Impacts of Building Construction Projects. Civil and Environmental Research, 3(1), 93-104.

Ikediashi, D. I. (2014). A Framework for Outsourcing Facilities Management Services in Nigeria's Public Hospitals. PhD thesis, School of the Built Environment Heriot-Watt University.

Ikediashi, D. I., Ogunlana, S. O., Oladokun, M. G., and Adewuyi, T. O. (2012). Assessing the Level of Commitment and Barriers to Sustainable Facilities Management Practice: A case of Nigeria. International Journal of Sustainable Built Environment, 1(2), 167-176. https://doi.org/10.1016/j.ijsbe.2013.06.002

Inkoom, E. E. (2013). Sustainability Adoption in Construction Organizations - An Institutional and Strategic Choice Perspective. MSc Thesis, Department of Building National University of Singapore.

Jolliffe, I.T. (2002). Principal component analysis. Springer.

Jones, M., and Saad, M. (2003). Managing Innovation in Construction. London: Thomas Telford. https://doi.org/10.1680/miic.30022

Joreskög, K. G., and Sörbom, D. (1993). LISREL 8: Structural equation modelling with SIMPLIS command language. Chicago: Scientific Software International.

Kaplan, R. S., \& Norton, D. P. (1992). The Balanced Scorecard: Measures that Drive Performance. Harvard Business Review, 70(1), 71-79.

Kazaz A, Manisali E, \& Ulubeyli S. (2008). Effect of Basic Motivational Factors on Construction Workforce Productivity in Turkey. J. Civil. Eng. Manage. 14, 95-106. https://doi.org/10.3846/1392-3730.2008.14.4

Kheni, N.A., and Akoogo, M.A. (2015). Determinants of Sustainable Construction Practices in Ghana Using Structural Equation Modelling. Journal of Sustainable Development; 8(3): 67-78. https://doi.org/10.5539/jsd.v8n3p67

Klang, A., Vikman, P.-A., and Brattebo, H. (2003). Sustainable Management of Demolition Waste, an Integrated Model for the Evaluation of Environmental, Economic and Social Aspects. Resources, Conservation and Recycling, 38(4), 317-334. https://doi.org/10.1016/S09213449(02)00167-2

Kline, R. B. (2005). Principles and Practice of Structural Equation Modelling, 2nd ed., The Guilford Press, New York, NY. 
Lee, K. H, Ahn, Y.H., Jeon, M., and Suh, M. J. (2014). Organisational Strategies to Support Sustainability in the Construction Company. World SB14, Barcelona.

Lilja, R. (2009). Negotiated Environmental Agreements in Promoting Material Efficiency in industry - First Steps in Finland. Journal of Cleaner Production, 17(9), 863-872. https://doi.org/10.1016/j.jclepro.2009.01.002

Linnenluecke, M. K., and Griffiths, A. (2010). Corporate Sustainability and Organisational Culture. Journal of World Business, 45(4), 357-366. https://doi.org/10.1016/j.jwb.2009.08.006

Liu, X., and Anbumozhi, V. (2009). Determinant Factors of Corporate Environmental Information Disclosure: An Empirical Study of Chinese listed companies. Journal of Cleaner Production, 17(6), 593-600. https://doi.org/10.1016/j.jclepro.2008.10.001

Maletič, M., Maletič, D., Jens J., Dahlgaard, J.J., Dahlgaard-Park, S.M., and, Gomišček, B. (2014). The Relationship between Sustainability-Oriented Innovation Practices and Organizational Performance: Empirical Evidence from Slovenian Organizations. Organizacija, 47(1): 3-13. https://doi.org/10.2478/orga-2014-0001

Mitchell, R. K., Agle, B. R., \& Wood, D. J. (1997). Toward a Theory of Stakeholder Identification and Salience: Defining the Principle of Who and What Really Counts. Academy of Management Review, 22(4), 853-886. https://doi.org/10.5465/amr.1997.9711022105

Molenaar, K., Washington, S., and Diekmann, J. (2000). Structural equation model of construction contract dispute potential. Journal of Engineering and Management, 126(4), 268-277. https://doi.org/10.1061/(ASCE)0733-9364(2000)126:4(268)

Moohammad, A. Y., Yusof Nor'Aini, Y. N., Ernawati M., \& Kamal, E. M. (2014). Influences of Firm Size, Age and Sector on Innovation Behaviour of Construction Consultancy Services Organizations in Developing Countries. Business Management Dynamics,4(4), 01-09.

OECD. (2005). Oslo Manual. Guidelines for Collecting and Interpreting Technological Innovation Data $3^{\text {rd }}$ ed., OECD Publishing. Available from: https://www.oecdilibrary.org/docserver/9789264013100-

en.pdf? expires $=1597908600 \& \mathrm{id}=\mathrm{id} \&$ accname $=$ guest $\&$ checksum=B731F8F8FBBD258D8108 E5776FA497E5

Parkin, S. (2000). Context and Drivers for Operationalising Sustainable Development, Proceedings of ICE, 138(6), 9-15. https://doi.org/10.1680/cien.2000.138.6.9

Porter, T. B. (2008). Managerial Applications of Corporate Social Responsibility and SystemsThinking for Achieving Sustainability Outcomes. Systems Research and Behavioural Science, 25, 397-411. https://doi.org/10.1002/sres.902

Pournara, I., and Wernisch, L. (2007). Factor analysis for gene regulatory networks and transcription factor activity profiles. BMC Bioinformatics, 8, Article number 61 (2007). https://doi.org/10.1186/1471-2105-8-61

Pujari, D. (2006). Eco-Innovation and New Product Development: Understanding the Influences on Market Performance. Technovation, 26(1), 76-85. https://doi.org/10.1016/j.technovation.2004.07.006

Roberts, R. W. (1992). Determinants of Corporate Social Responsibility Disclosure: An Application of Stakeholder Theory. Accounting, Organizations and Society, 17(6), 595-612. https://doi.org/10.1016/0361-3682(92)90015-K

Santos, J. B., and Brito, L. A. L. (2012). Toward a Subjective Measurement Model for Firm Performance. BAR, Braz. Adm. Rev., 9, no. spe Rio de Janeiro May 2012, 95-117. https://doi.org/10.1590/S1807-76922012000500007

Sharma, S. (2002). Research in Corporate Sustainability: What really matters. Research in Corporate Sustainability: The Evolving Theory and Practice of Organizations in the Natural Environment, 1-29.

Shen, L. Y., Vivian, W. Y. T., Leona, T., and Ji, Y. B. (2010). Project Feasibility Study: the key to Successful Implementation of Sustainable and Socially Responsible Construction Management Practice. Journal of Cleaner Production, 18, 254-259. https://doi.org/10.1016/j.jclepro.2009.10.014

Shumacker, R., and Lomax, R. (2004). A beginner's guide to structural equation modelling, 2nd edition. Lawrence Erlbaum associates. 
Sommerville, J., and Craig, N. (2006). Implementing IT in Construction. London: Routledge. https://doi.org/10.4324/9780203030394

Tabachnick, B. G., and Fidell, L. S. (2007). Using multivariate statistics (5th edition). Allyn \& Bacon/Pearson Education.

Tam, W. Y. V. (2008). On the Effectiveness of Implementing a Waste Management-Plan Method in Construction. Waste Management, 28, 1072-1080.

Uadiale, O. M., and Fagbemi, T. O. (2012). Corporate Social Responsibility and Financial Performance in Developing Economies: The Nigerian Experience. Journal of Economics and Sustainable Development, 3(4), 44-54.

Ugochukwu, C. N. C. (2008). Sustainable Environmental Management in the Niger Delta Region of Nigeria: Effects of Hydrocarbon Pollution on Local Economy. PhD Thesis, Brandenburg University of Technology.

Ujene, A. O. (2014). Integrating Environmental Priority Concerns in Building Planning and Production in Niger Delta, Nigeria. Journal of Architecture, Planning and Construction Management, 4(2), 36-56.

Ujene, A. O., and Oladokun, M. G. (2017). Sustainability Assessment in Construction Organisations' Project Delivery Practice in Nigeria, J. Building Performance, 8(1), 12-24.

Waddock, S. A., and Graves, S. B. (1997). The Corporate Social Performance-Financial Performance Link. Strategic Management Journal, 18(4), 303-319. https://doi.org/10.1002/(SICI)1097-0266(199704)18:4<303::AID-SMJ869>3.0.CO;2-G

Waziri, A. G. Nor'Aini Yusof, N., and Osmadi, A. (2015). Green Construction Practices (GCP) Implementation in Nigeria: How Far So Far? Advances in Environmental Biology, 9(5), 84-86.

Weshah, S. R., Dahiyat, D. A., Awwad, M. A., and Hajjat, E. S. (2012). The Impact of Adopting Corporate Social Responsibility on Corporate Financial Performance: Evidence from Jordanian Banks. Interdisciplinary Journal of Contemporary Research in Business, 4(5), 34-44.

Widen, K. (2003). Encouraging Innovation through New Approaches to Procurement. In B. Atkin, J. Borgbrant \& P. E. Josephson, (Eds.) Construction process improvement. Oxford: Blackwell science, 143-151. https://doi.org/10.1002/9780470690697.ch12

World Business Council for Sustainable Development (WBCSD). (2002). Sustainable Development Reporting: Striking the Balance.

Yamane, T. (1967). Statistics: An Introductory Analysis2nd ed. New York: Harper and Row.

Zulu, S. L. (2007). The impact of project management process quality on construction project performance: a structural equation model. Unpublished $\mathrm{PhD}$ thesis, Heriot Watt University, Edinburgh.

\section{AUTHORS' SHORT BIOGRAPHIES}

Dr Monday Otali is a Senior Lecturer at the Department of Building, Faculty of Environmental Studies, University of Uyo, Nigeria. Dr Monday Otali is a Construction Management Expert. His research interest includes sustainability in construction and construction management. He obtained his $\mathrm{PhD}$ degree in Construction Management in 2018 from the University of Uyo. He also obtained his Master degree (MSc in Construction Management) in 2011 from the University of Jos. Dr Monday Otali graduated with BSc (Hons) degree in Building from the Department of Building, University of Jos in 2006. He is a corporate member of the Nigerian Institute of Building and a registered and certified member of the Council of Registered Builders of Nigeria.

E-mail: otalimonday@yahoo.com

Dr Godwin Idoro is a Professor of Project Management at the Department of Building, Faculty of Environmental Sciences, University of Lagos, Nigeria. He obtained his $\mathrm{PhD}$ degree in Building in 2008 from the University of Lagos. He was the Head of the Department of Building, University of Lagos (2011-2015). He is a fellow of the Nigerian Institute of Quantity Surveying and a corporate member of the Nigerian Institute of Building. His current research is on project team integration in the construction industry in Nigeria. He has successfully supervised ten (10) PhDs.

E- mail: iroroidoro@yahoo.com 
Dr Godfrey Udo is a Professor of Estate Management at the Department of Estate Management, University of Uyo, Nigeria. He graduated with a degree in Urban Estate Surveying at Nottingham Trent University in 1980. He obtained his MSc in Estate Management in 1987 and a PhD in Estate Management in 1991. He was the Head of the Department of Estate Management, University of Uyo in 2008, and the Dean of the Faculty of Environmental Studies in 2012.He was appointed Dean of Post-Graduate School in 2016. He was also appointed Deputy Vice-Chancellor (Administration) in 2017. He has published articles in both international and local journals. He has also presented papers in both local and international conferences. He is a fellow of the Nigerian Institution of Estate Surveyors and Valuers and a registered member of the Estate Surveyors and Valuers Registration Board of Nigeria.

E-mail: godfrey.u2@gmail.com

ORCID iD: https://orcid.org/0000-0002-6985-8297 